\title{
A boundary integral equation with the generalized Neumann kernel for a mixed boundary value problem in unbounded multiply connected regions
}

\author{
Samer AA Al-Hatemi ${ }^{1}$, Ali HM Murid ${ }^{1,2^{*}}$ and Mohamed MS Nasser ${ }^{3,4}$
}

${ }^{*}$ Correspondence:

alihassan@utm.my

${ }^{1}$ Department of Mathematical

Sciences, Faculty of Science,

Universiti Teknologi Malaysia, Johor

Bahru, Johor 81310 UTM, Malaysia

${ }^{2}$ UTM Centre for Industrial and

Applied Mathematics, Universiti

Teknologi Malaysia, Johor Bahru,

Johor 81310 UTM, Malaysia

Full list of author information is

available at the end of the article

\begin{abstract}
In this paper we propose a new method for solving the mixed boundary value problem for the Laplace equation in unbounded multiply connected regions. All simple closed curves making up the boundary are divided into two sets. The Dirichlet condition is given for one set and the Neumann condition is given for the other set. The mixed problem is reformulated in the form of a Riemann-Hilbert $(\mathrm{RH})$ problem which leads to a uniquely solvable Fredholm integral equation of the second kind. Three numerical examples are presented to show the effectiveness of the proposed method.
\end{abstract}

Keywords: mixed boundary value problem; $\mathrm{RH}$ problem; Fredholm integral equation; generalized Neumann kernel

\section{Introduction}

In the present paper, we continue the research concerned with the study of mixed boundary value problems in the plane started in [1]. We consider a mixed boundary value problem for the Laplace equation in an unbounded multiply connected regions. Recently, the interplay of the RH boundary value problem and integral equations with the generalized Neumann kernel on unbounded multiply connected regions has been investigated in [2]. Based on the reformulations of the Dirichlet problem, the Neumann problem and conformal mappings as RH problems, boundary integral equations with the generalized Neumann kernel have been implemented successfully in [3] to solve the Dirichlet problem and the Neumann problem and in [4-6] to compute the conformal mappings of unbounded multiply connected regions onto the classical canonical slit domains.

The mixed boundary value problem also can be reformulated as an $\mathrm{RH}$ problem (see, e.g., [7-9]). Recently, Nasser et al. [1] have presented a uniquely solvable boundary integral equation with the generalized Neumann kernel for solving the mixed boundary value problem in bounded multiply connected regions. The idea of this paper is to reformulate the mixed boundary value problem to the form of the RH problem in unbounded multiply connected regions. Based on this reformulation, we present a new boundary integral equation method for two-dimensional Laplace's equation with the mixed boundary condition

(c) 2013 Al-Hatemi et al.: licensee Springer. This is an Open Access article distributed under the terms of the Creative Commons Attribution License (http://creativecommons.org/licenses/by/2.0), which permits unrestricted use, distribution, and reproduction in any medium, provided the original work is properly cited. 
in unbounded multiply connected regions. The method is based on a uniquely solvable boundary integral equation with the generalized Neumann kernel.

This paper is organized as follows. After presenting some auxiliary materials in Section 2, we present in Section 3 the mixed boundary value problem in unbounded multiply connected regions. In Section 4, we give an explanation of an integral equation with the generalized Neumann kernel and its solvability. The reduction of the mixed boundary value problem to the form of the RH problem is given in Section 5. In Section 6, we present the solution of the mixed boundary problem via an integral equation method. In Section 7, we explain briefly the numerical implementation of the method. In Section 8, we illustrate the method by presenting two numerical examples with exact solutions and also one example without an exact solution.

\section{Notations and auxiliary material}

In this section, we review some properties of the generalized Neumann kernel from [2, 3, $5,10]$.

We consider an unbounded multiply connected region $G$ of connectivity $m \geq 2$ with boundary $\Gamma=\partial G=\bigcup_{j=1}^{m} \Gamma_{j}$ consisting of $m$ clockwise oriented smooth closed Jordan curves $\Gamma_{j}, j=1,2, \ldots, m$. The complement $G^{-}:=\overline{\mathbb{C}} \backslash \bar{G}$ consists of $m$ bounded simply connected components $G_{j}$ interior to $\Gamma_{j}, j=1,2, \ldots, m$. We assume $\infty \in G, 0 \in G$ (see Figure 1 ).

The curves $\Gamma_{j}$ are parametrized by $2 \pi$-periodic twice continuously differentiable complex-valued functions $\eta_{j}(t)$ with non-vanishing first derivatives, i.e.,

$$
\dot{\eta}_{j}(t)=d \eta_{j}(t) / d t \neq 0, \quad t \in J_{j}:=[0,2 \pi], j=1,2, \ldots, m .
$$

The total parameter domain $J$ is the disjoint union of the intervals $J_{j}, j=1,2, \ldots, m$. We define a parametrization of the whole boundary $\Gamma$ as the complex-valued function $\eta$ defined on $J$ by

$$
\eta(t):= \begin{cases}\eta_{1}(t), & t \in J_{1}, \\ \vdots & \\ \eta_{m}(t), & t \in J_{m} .\end{cases}
$$

Let $H$ be the space of all real Hölder continuous functions on the boundary $\Gamma$. In view of the smoothness of $\eta$, a function $\phi \in H$ can be interpreted via $\hat{\phi}(t):=\phi(\eta(t)), t \in J$, as a real Hölder continuous $2 \pi$-periodic function $\hat{\phi}(t)$ of the parameter $t \in J$, i.e.,

$$
\hat{\phi}(t):= \begin{cases}\hat{\phi}_{1}(t), \quad t \in J_{1}, \\ \vdots & \\ \hat{\phi}_{m}(t), & t \in J_{m},\end{cases}
$$

Figure 1 An unbounded multiply connected region $G$ of connectivity $m$.

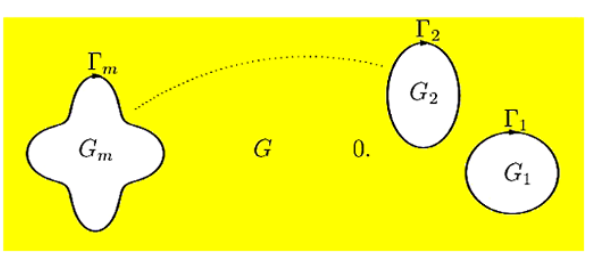


with real Hölder continuous $2 \pi$-periodic functions $\hat{\phi}_{j}$ defined on $J_{j}$. So, here and in what follows, we do not distinguish between functions of the form $\psi(\eta(t))$ and $\psi(t)$.

The subspace of $H$ which consists of all piecewise constant functions defined on $\Gamma$ is denoted by $S$, i.e., a function $h \in S$ has the representation

$$
h(t):= \begin{cases}h_{1}, & t \in J_{1}, \\ \vdots & \\ h_{m}, & t \in J_{m},\end{cases}
$$

where $h_{1}, \ldots, h_{m}$ are real constants. For simplicity, the function $h$ is denoted by

$$
h(t)=\left(h_{1}, \ldots, h_{m}\right)
$$

\section{The mixed boundary value problem}

Let $S_{\mathbf{d}}$ and $S_{\mathbf{n}}$ be two subsets of the set $\{1, \ldots, m\}$ such that

$$
S_{\mathbf{d}} \neq \emptyset, \quad S_{\mathbf{n}} \neq \emptyset, \quad S_{\mathbf{d}} \cup S_{\mathbf{n}}=\{1, \ldots, m\} \quad \text { and } \quad S_{\mathbf{d}} \cap S_{\mathbf{n}}=\emptyset .
$$

Let $\mathbf{n}$ be the exterior unit normal to $\Gamma$ and let $\phi \in H$ be a given function. We consider the mixed boundary value problem

$$
\begin{aligned}
& \Delta u(z)=0, \quad z \in G, \\
& u(\eta(t))=\phi_{j}(t), \quad t \in J, j \in S_{\mathbf{d}}, \\
& \frac{\partial u(\eta(t))}{\partial \mathbf{n}}=\phi_{j}(t), \quad t \in J, j \in S_{\mathbf{n}},
\end{aligned}
$$

for a real function $u$ in G. We call (6b) and (6c) Dirichlet conditions and Neumann conditions, respectively.

Problem (6a)-(6c) reduces to the Dirichlet problem for $S_{\mathbf{n}}=\emptyset$ and to the Neumann problem for $S_{\mathbf{d}}=\emptyset$. Both problems have been considered in [3]. So, we assume in this paper that $S_{\mathbf{n}} \neq \emptyset$ and $S_{\mathbf{d}} \neq \emptyset$.

The mixed boundary value problem (6a)-(6c) is uniquely solvable [11]. Its unique solution $u$ can be regarded as a real part of an analytic function $F$ in $G$ which is not necessary single-valued. The function $F$ can be written as

$$
F(z)=f(z)-\sum_{j=1}^{m} a_{j} \log \left(z-z_{j}\right)
$$

where $f$ is a single-valued analytic function in $G, z_{j}$ are fixed points in $G_{j}, j=1,2, \ldots, m$; and $a_{1}, \ldots, a_{m}$ are real constants uniquely determined by $\phi$ (see [12]). Without lost of generality, we assume that $\operatorname{Im} f(\infty)=0$. The constants $a_{1}, \ldots, a_{m}$ are chosen to ensure that (see [12, p.149] and [3])

$$
\int_{\Gamma_{j}} f^{\prime}(\eta) d \eta=0, \quad j=1,2, \ldots, m
$$


i.e., $a_{j}$ are given by (see [3])

$$
a_{j}=\frac{1}{2 \pi \mathrm{i}} \int_{\Gamma_{j}} F^{\prime}(\eta) d \eta, \quad j=1,2, \ldots, m .
$$

The constants $a_{j}$ satisfy

$$
\sum_{j=1}^{m} a_{j}=\sum_{j=1}^{m} \frac{1}{2 \pi \mathrm{i}} \int_{\Gamma_{j}} F^{\prime}(\eta) d \eta=\frac{1}{2 \pi \mathrm{i}} \int_{\Gamma} F^{\prime}(\eta) d \eta=0 .
$$

\section{Integral equation}

In this paper we assume that the function $A$ is a continuously differentiable complexvalued function given by

$$
A(t):=e^{-\mathrm{i} \theta(t)}
$$

where $\theta$ is the real piecewise constant function

$$
\theta(t)=\left(\theta_{1}, \ldots, \theta_{m}\right)
$$

with either $\theta_{j}=0$ or $\theta_{j}=\pi / 2, j=1, \ldots, m$. Here the function $A(t)$ is different from the ones used in $[1,3]$. The generalized Neumann kernel formed with $A$ and $\eta(t)$ is defined by

$$
N(s, t):=\frac{1}{\pi} \operatorname{Im}\left(\frac{A(s)}{A(t)} \frac{\dot{\eta}(t)}{\eta(t)-\eta(s)}\right) .
$$

We also define a real kernel $M$ by

$$
M(s, t):=\frac{1}{\pi} \operatorname{Re}\left(\frac{A(s)}{A(t)} \frac{\dot{\eta}(t)}{\eta(t)-\eta(s)}\right) .
$$

The kernel $N$ is continuous and the kernel $M$ has a cotangent singularity type (see [2] for more details). Hence, the operator

$$
\mathbf{N} \mu(s):=\int_{J} N(s, t) \mu(t) d t, \quad s \in J,
$$

is a Fredholm integral operator and the operator

$$
\mathbf{M} \mu(s):=\int_{J} M(s, t) \mu(t) d t, \quad s \in J
$$

is a singular integral operator.

The solvability of boundary integral equations with the generalized Neumann kernel is determined by the index (the change of the argument of $A$ on the curves $\Gamma_{j}$ divided by $2 \pi$ ) of the function $A$ (see [2]). For the function $A$ given by (10), the indices $\kappa_{j}$ of $A$ on the curves $\Gamma_{j}$ and the index $\kappa=\sum_{j=1}^{m} \kappa_{j}$ of $A$ on the whole boundary curve $\Gamma$ are given by

$$
\kappa_{j}=0, \quad j=1, \ldots, m, \quad \kappa=0 .
$$


The generalized Neumann kernel for an integral equation associated with the mixed boundary value problem which will be presented in this paper is different from the generalized Neumann kernel for the integral equation considered in [1,3]. Thus, not all of the properties of the generalized Neumann kernel which have been studied in [3] are valid for the generalized Neumann kernel which will be studied in this paper. For example, it is still true that $\lambda=1$ is not an eigenvalue of the generalized Neumann kernel which means that the presented integral equation is uniquely solvable.

By using the same approach used in [3] for unbounded multiply connected regions, we can prove that the properties of the generalized Neumann kernel proved in [3], except Theorem 8, Theorem 10 and Corollary 2, are still valid for the generalized Neumann kernel formed with the function $A(t)$ in (10) above (see [5]).

Thus, we have from [5] the following theorem (see also $[2,10])$.

Theorem 1 For a given function $\gamma \in H$, there exist unique functions $h \in S$ and $\mu \in H$ such that

$$
A g=\gamma+h+\mathrm{i} \mu
$$

are boundary values of a unique analytic function $g(z)$ in $G$ with $g(\infty)=0$. The function $\mu$ is the unique solution of the integral equation

$$
(\mathbf{I}-\mathbf{N}) \mu=-\mathbf{M} \gamma
$$

and the function $h$ is given by

$$
h=[\mathbf{M} \mu-(\mathbf{I}-\mathbf{N}) \gamma] / 2 .
$$

\section{Reformulation of the mixed boundary value problem as an RH problem}

The mixed boundary value problem can be reduced to an RH problem as follows. Let the boundary values of the multi-valued analytic function $F$ be given by

$$
F=\psi+\mathrm{i} \varphi
$$

Although, the function $F(z)$ is in general multi-valued, its derivative $F^{\prime}$ is a single-valued analytic function on $G$. The boundary values of the function $F^{\prime}(z)$ are given by

$$
\dot{\eta} F^{\prime}=\psi^{\prime}+\mathrm{i} \varphi^{\prime} .
$$

For the Dirichlet conditions, i.e., $t \in J_{j}$ and $j \in S_{\mathbf{d}}$, the functions $\psi_{j}$ are equal to the known functions $\phi_{j}(t)$ (see $\left.(6 \mathrm{~b})\right)$. Thus, the function $F(z)$ satisfies the RH problem

$$
\operatorname{Re}\left[F\left(\eta_{j}(t)\right)\right]=\phi_{j}(t), \quad t \in J_{j}, j \in S_{\mathbf{d}}
$$

The Neumann conditions can also be reduced to an RH problem by using the CauchyRiemann equations and integrating along the boundaries $\Gamma_{j}, j \in S_{\mathbf{n}}$. Let $\mathbf{T}(\zeta)$ be the unit 
tangent vector and $\mathbf{n}(\zeta)$ be the unit external normal vector to $\Gamma$ at $\zeta \in \Gamma$. Let also $v(\zeta)$ be the angle between the normal vector $\mathbf{n}(\zeta)$ and the positive real axis, i.e., $\mathbf{n}(\zeta)=e^{\mathrm{i} v(\zeta)}$. Then

$$
e^{\mathrm{i} v(\eta(t))}=-\mathrm{i} \mathbf{T}(\eta(t))=-\mathrm{i} \frac{\dot{\eta}(t)}{|\dot{\eta}(t)|}
$$

Thus,

$$
\frac{\partial u}{\partial \mathbf{n}}=\nabla u \cdot \mathbf{n}=\cos v \frac{\partial u}{\partial x}+\sin v \frac{\partial u}{\partial y}=\operatorname{Re}\left[e^{\mathrm{i} v}\left(\frac{\partial u}{\partial x}-\mathrm{i} \frac{\partial u}{\partial y}\right)\right]
$$

Since $u(z)=\operatorname{Re} F(z)$, then by the Cauchy-Riemann equations, we have

$$
F^{\prime}(z)=\frac{\partial u(z)}{\partial x}-\mathrm{i} \frac{\partial u(z)}{\partial y}
$$

Thus, the function $F^{\prime}(z)$ satisfies the RH problem

$$
\operatorname{Re}\left[-\mathrm{i} \dot{\eta}_{j}(t) F^{\prime}\right]=\left|\dot{\eta}_{j}(t)\right| \frac{\partial u}{\partial \mathbf{n}}, \quad t \in J_{j}, j \in S_{\mathbf{n}} .
$$

If we define the real piecewise constant function

$$
\theta(t)= \begin{cases}0, & t \in J_{j}, j \in S_{\mathbf{d}} \\ \pi / 2, & t \in J_{j}, j \in S_{\mathbf{n}}\end{cases}
$$

the boundary values of the function $F(z)$ satisfy on the boundary $\Gamma$ the condition

$$
\operatorname{Re}\left[e^{-\mathrm{i} \theta(t)} F(\eta(t))\right]=\hat{\phi}(t),
$$

where

$$
\hat{\phi}(t)= \begin{cases}\phi_{j}(t), & t \in J_{j}, j \in S_{\mathbf{d}}, \\ \varphi_{j}(t), & t \in J_{j}, j \in S_{\mathbf{n}}\end{cases}
$$

is known and

$$
\varphi_{j}^{\prime}(t)=\operatorname{Re}\left[-\mathrm{i} \dot{\eta}_{j}(t) F^{\prime}\left(\eta_{j}(t)\right)\right]=\phi_{j}(t)\left|\dot{\eta}_{j}(t)\right|, \quad t \in J_{j}, j \in S_{\mathbf{n}}
$$

The functions $\phi_{j}(t)$ for $j \in S_{\mathbf{d}} \cup S_{\mathbf{n}}$ are given by (6b) and (6c). The functions $\varphi_{j}(t)$ can be then computed for $t \in J_{j}$ and $j \in S_{\mathbf{n}}$ by integrating the functions $\varphi_{j}^{\prime}(t)$. Then it follows from (7), (26) and (27) that the function $f(z)$ is a solution of the $\mathrm{RH}$ problem

$$
\operatorname{Re}\left[e^{-\mathrm{i} \theta(t)} f(\eta(t))\right]=\hat{\phi}(t)+\sum_{k=1}^{m} a_{k} \operatorname{Re}\left[e^{-\mathrm{i} \theta(t)} \log \left(\eta(t)-z_{k}\right)\right]
$$

or briefly,

$$
\operatorname{Re}\left[e^{-\mathrm{i} \theta(t)} f(\eta(t))\right]=\hat{\phi}(t)+\sum_{k \in S_{\mathbf{n}}} a_{k} \gamma^{[k]}(t)+\sum_{k \in S_{\mathbf{d}}} a_{k} \gamma^{[k]}(t)
$$


where

$$
\gamma^{[k]}(t)=\operatorname{Re}\left[e^{-\mathrm{i} \theta(t)} \log \left(\eta(t)-z_{k}\right)\right]
$$

for $k=1,2, \ldots, m$. In view of (8) and (28), the real constants $a_{k}$ are known for $k \in S_{\mathbf{n}}$ and are given by

$$
a_{k}=\frac{1}{2 \pi} \int_{J_{k}} \phi_{k}(t)\left|\dot{\eta}_{k}(t)\right| d t, \quad k \in S_{\mathbf{n}} .
$$

However, for $k \in S_{\mathbf{d}}$, the real constants $a_{k}$ are unknown. Thus, the boundary condition (29) can be written as

$$
\operatorname{Re}\left[e^{-\mathrm{i} \theta(t)} f(\eta(t))\right]=\hat{\psi}(t)+\sum_{k \in S_{\mathbf{d}}} a_{k} \gamma^{[k]}(t), \quad t \in J
$$

where the function $\hat{\psi}(t)$ is known and is given by

$$
\hat{\psi}(t)= \begin{cases}\phi_{j}(t)+\sum_{k \in S_{\mathbf{n}}} a_{k} \gamma_{j}^{[k]}(t), \quad t \in J_{j}, j \in S_{\mathbf{d}}, \\ \varphi_{j}(t)+\sum_{k \in S_{\mathbf{n}}} a_{k} \gamma_{j}^{[k]}(t), \quad t \in J_{j}, j \in S_{\mathbf{n}} .\end{cases}
$$

Obviously, the functions $\hat{\psi}_{j}(t)$ are known explicitly for $t \in J_{j}$ with $j \in S_{\mathbf{d}}$. However, for $t \in J_{j}$ with $j \in S_{\mathbf{n}}$, it is required to integrate $\varphi_{j}^{\prime}(t)$ to obtain $\varphi_{j}(t)$.

The functions $\varphi_{j}(t)$ are not necessary $2 \pi$-periodic. In order to keep dealing with periodic functions numerically, we do not compute $\varphi_{j}(t)$ directly by integrating the functions $\varphi_{j}^{\prime}(t)$. Instead, we integrate the functions

$$
\hat{\psi}_{j}^{\prime}(t)=\phi_{j}(t)\left|\dot{\eta}_{j}(t)\right|+\sum_{k \in S_{\mathbf{n}}} a_{k} \frac{d}{d t} \gamma_{j}^{[k]}(t)
$$

According to the definitions of the constants $a_{k}$ and the functions $\gamma^{[k]}$, we have

$$
\int_{0}^{2 \pi} \hat{\psi}_{j}^{\prime}(t) d t=0
$$

which implies that the functions $\hat{\psi}_{j}(t)=\varphi_{j}(t)+\sum_{k \in S_{\mathbf{n}}} a_{k} \gamma_{j}^{[k]}(t)$ are always $2 \pi$-periodic. By using the Fourier series for $t \in J_{j}$ with $j \in S_{\mathbf{n}}$, the functions $\hat{\psi}_{j}^{\prime}(t)$ can be written as

$$
\hat{\psi}_{j}^{\prime}(t)=\sum_{i=1}^{\infty} a_{i}^{[j]} \cos i t+\sum_{i=1}^{\infty} b_{i}^{[j]} \sin i t
$$

Then the functions $\hat{\psi}_{j}(t)$ are given for $t \in J_{j}$ with $j \in S_{\mathbf{n}}$ by

$$
\hat{\psi}_{j}(t)=\tilde{\psi}_{j}(t)+c_{j}
$$

where $c_{j}$ are undetermined real constants and the functions $\tilde{\psi}_{j}(t)$ are given by

$$
\tilde{\psi}_{j}(t)=\sum_{i=1}^{\infty} \frac{a_{i}^{[j]}}{i} \sin i t-\sum_{i=1}^{\infty} \frac{b_{i}^{[j]}}{i} \cos i t, \quad t \in J_{j}, j \in S_{\mathbf{n}} .
$$


Hence, the boundary condition (33) can then be written as

$$
\operatorname{Re}\left[e^{-\mathrm{i} \theta(t)} f(\eta(t))\right]=\hat{\gamma}(t)+\tilde{h}(t)+\sum_{k \in S_{\mathbf{d}}} a_{k} \gamma^{[k]}(t), \quad t \in J,
$$

where $\tilde{h}(t)$ is the real piecewise constant function

$$
\tilde{h}(t)= \begin{cases}0, & t \in J_{j}, j \in S_{\mathbf{d}}, \\ c_{j}, & t \in J_{j}, j \in S_{\mathbf{n}},\end{cases}
$$

and the function $\hat{\gamma}(t)$ is given by

$$
\hat{\gamma}(t)= \begin{cases}\phi_{j}(t)+\sum_{k \in S_{\mathbf{n}}} a_{k} \gamma_{j}^{[k]}(t), & t \in J_{j}, j \in S_{\mathbf{d}}, \\ \tilde{\psi}_{j}(t), & t \in J_{j}, j \in S_{\mathbf{n}}\end{cases}
$$

Let $c:=f(\infty)$ (unknown real constant) and $g(z)$ be the analytic function defined on $G$ by

$$
g(z):=f(z)-c, \quad z \in G .
$$

Then $g(z)$ is analytic on $G$ with $g(\infty)=0$. The function $g(z)$ is a solution of the RH problem

$$
\operatorname{Re}[A(t) g(\eta(t))]=\tilde{\gamma}(t)+h(t)+\sum_{j \in S_{\mathbf{d}}} a_{j} \gamma^{[j]}(t), \quad t \in J,
$$

where the function $A(t)$ is given by (10) and the function $h(t)$ is defined by

$$
h(t)=\hat{h}(t)-c \cos \theta(t), \quad t \in J .
$$

\section{The solution of the mixed boundary value problem}

Let $\mu(t):=\operatorname{Im}[A(t) g(\eta(t))]$. Then the boundary values of the function $g(z)$ are given on the boundary $\Gamma$ by

$$
A(t) g(\eta(t))=\hat{\gamma}(t)+h(t)+\sum_{j \in S_{\mathbf{d}}} a_{j} \gamma^{[j]}(t)+\mathrm{i} \mu(t), \quad t \in J,
$$

where $\hat{\gamma}(t), \gamma^{[1]}, \ldots, \gamma^{[m]}$ are knowns and $h, \mu$ are unknowns. The real constants $a_{j}$ are known for $j \in S_{\mathbf{n}}$ and unknown for $j \in S_{\mathbf{d}}$.

For $j \in S_{\mathbf{d}}$, let the functions $\hat{\mu}$ and $\mu^{[j]}$ be the unique solutions of the integral equations

$$
(\mathbf{I}-\mathbf{N}) \hat{\mu}=-\mathbf{M} \hat{\gamma}, \quad(\mathbf{I}-\mathbf{N}) \mu^{[j]}=-\mathbf{M} \gamma^{[j]},
$$

respectively, $\hat{h}(t)$ and $h^{[j]}$ be given by

$$
\hat{h}(t)=[\mathbf{M} \hat{\mu}-(\mathbf{I}-\mathbf{N}) \hat{\gamma}] / 2, \quad h^{[j]}=\left[\mathbf{M} \mu^{[j]}-(\mathbf{I}-\mathbf{N}) \gamma^{[j]}\right] / 2 .
$$

Then it follows from Theorem 1 that

$$
A(t) \hat{g}(\eta(t))=\hat{\gamma}+\hat{h}+\mathrm{i} \hat{\mu}+\sum_{j \in S_{\mathbf{d}}} a_{j}\left(\gamma^{[j]}+h^{[j]}+\mathrm{i} \mu^{[j]}\right)
$$


are boundary values of an analytic function $\hat{g}(z)$. By the uniqueness of the functions $h$ and $\mu$ in (44), it follows from (44) and (47) that

$$
\mu(t)=\hat{\mu}+\sum_{j \in S_{\mathbf{d}}} a_{j} \mu^{[j]},
$$

and

$$
h(t)=\hat{h}+\sum_{j \in S_{\mathbf{d}}} a_{j} h^{[j]}
$$

Equation (49a) with the following equation (from (9)),

$$
\sum_{j \in S_{\mathbf{d}}} a_{j}=-\sum_{j \in S_{\mathbf{n}}} a_{j}
$$

represents a linear system of $m$ equations. Since from (43) the function $h(t)$ is given by

$$
h(t)= \begin{cases}-c, & t \in J_{j}, j \in S_{\mathbf{d}}, \\ c_{j}, & t \in J_{j}, j \in S_{\mathbf{n}},\end{cases}
$$

only the constants $c, a_{j}$ for $j \in S_{\mathbf{d}}$ and $c_{j}$ for $j \in S_{\mathbf{n}}$ are unknowns. Thus, linear equations (49a) and (49b) represent a linear system of $m+1$ equations in $m+1$ unknowns $a_{j}$ for $j \in S_{\mathbf{d}}$ and $c_{j}$ for $j \in S_{\mathbf{n}}$.

By obtaining the values of the constants $a_{j}$, we obtain the functions $\mu$ from (48) and $h$ from (49a). Consequently, the boundary values of the function $g$ are given by

$$
A(t) g(\eta(t))=\gamma(t)+h(t)+\mathrm{i} \mu(t), \quad t \in J,
$$

where

$$
\gamma(t)=\hat{\gamma}(t)+\sum_{j \in S_{\mathbf{d}}} a_{j} \gamma^{[j]}(t), \quad t \in J
$$

The function $g(z)$ can be computed for $z \in G$ by the Cauchy integral formula. Then the function $f(z)$ is computed from

$$
f(z)=c+g(z)
$$

Finally, the solution of the mixed boundary value problem can be computed from $u(z)=$ $\operatorname{Re} F(z)$, where $F(z)$ is given by (7).

\section{Numerical implementations}

Since the functions $A_{j}$ and $\eta_{j}$ are $2 \pi$-periodic, the integrals in the operators $\mathbf{N}$ and $\mathbf{M}$ in integral equations (45) are best discretized on an equidistant grid by the trapezoidal rule [13]. The computational details are similar to previous works in $[4,5,10,14]$. For analytic integrands, the rate of convergence is better than $1 / n^{k}$ for any positive integer $k$ (see, e.g., $[15, \mathrm{p} .83])$. The obtained approximate solutions of the integral equations converge to 
the exact solutions with a similarly rapid rate of convergence (see, e.g., [13, p.322]). Since the smoothness of the integrands in (45) depends on the smoothness of the function $\eta(t)$, results of high accuracy can be obtained for very smooth boundaries.

By using the trapezoidal rule with $n$ (an even positive integer) equidistant collocation points on each boundary component, solving integral equations (45) reduces to solving $m n$ by $m n$ linear systems. Since integral equations (45) are uniquely solvable, then for sufficiently large values of $n$, the obtained linear systems are also uniquely solvable [13].

In this paper, the linear systems are solved using the Gauss elimination method. By solving the linear systems, we obtain approximations to $\hat{\mu}$ and $\mu^{[j]}$ for $j \in S_{\mathbf{d}}$, which give approximations to $\hat{h}$ and $h^{[j]}$ for $j \in S_{\mathbf{d}}$ from (46). By solving (49a) and (49b), we get approximations to the constants $c, a_{j}$ for $j \in S_{\mathbf{d}}$ and $c_{j}$ for $j \in S_{\mathbf{n}}$. These give approximations to the boundary values of the function $g(z)$ from (50). Then the values of $g(z)$ for $z \in G$ are calculated by the Cauchy integral formula. For points $z$ which are not close to the boundary $\Gamma$, the integrals in the Cauchy integral formula are approximated by the trapezoidal rule. However, for points $z$ near the boundary $\Gamma$, the integrand is nearly singular. For the latter case, the integral in the Cauchy integral formula can be calculated accurately using the method suggested in [16, Eq. (23)]. Then approximate values of the function $f(z)$ are computed from (52). Finally, in view of (7), the approximate solution of the mixed boundary value problem can be computed from

$$
u(z)=\operatorname{Re} F(z)=\operatorname{Re} f(z)-\sum_{j=1}^{m} a_{j} \log \left|z-z_{j}\right| .
$$

In this paper, we have considered regions with smooth boundaries. For some ideas on how to solve numerically boundary integral equations with the generalized Neumann kernel on regions with piecewise smooth boundaries, see [14].

\section{Numerical examples}

In this section, the proposed method is used to solve three mixed boundary value problems in unbounded multiply connected regions with smooth boundaries.

Example 1 In this example, we consider an unbounded multiply connected region of connectivity 4 bounded by the four circles (see Figure 2)

$$
\begin{aligned}
& \Gamma_{1}: \eta_{1}(t)=3+0.5 \mathrm{i}+0.5 e^{-\mathrm{i} t}, \\
& \Gamma_{2}: \eta_{2}(t)=1+\mathrm{i}+0.5 e^{-\mathrm{i} t}, \\
& \Gamma_{3}: \eta_{3}(t)=1-\mathrm{i}+0.5 e^{-\mathrm{i} t}, \\
& \Gamma_{4}: \eta_{4}(t)=-1+0.5 e^{-\mathrm{i} t},
\end{aligned}
$$

where $0 \leq t \leq 2 \pi$.

We assume that the conditions on the boundaries $\Gamma_{1}, \Gamma_{2}$ are the Neumann conditions and the conditions on the boundaries $\Gamma_{3}, \Gamma_{4}$ are the Dirichlet conditions. The functions $\phi_{j}$ in (6b)-(6c) are obtained based on choosing an exact solution of the form

$$
u(z)=\operatorname{Re} \frac{1}{z-i-1} .
$$




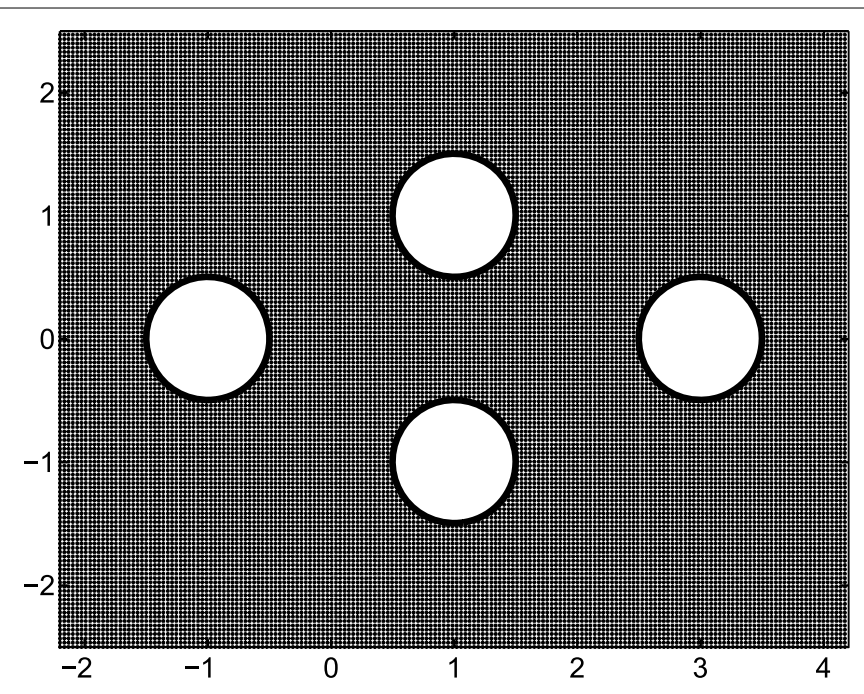

Figure 2 The region for Example 1.

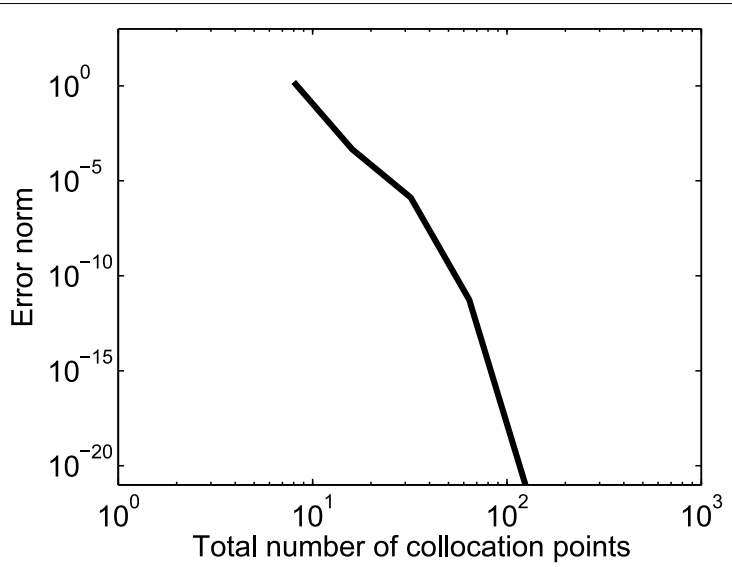

Figure 3 The error norm (54) for Example 1.

We use the error norm

$$
\int_{0}^{2 \pi}\left|u\left(1+\mathrm{i}+0.5 e^{-\mathrm{i} t}\right)-u_{n}\left(1+\mathrm{i}+0.5 e^{-\mathrm{i} t}\right)\right|^{2} d t
$$

where $u(z)$ is the exact solution of the mixed boundary value problem and $u_{n}(z)$ is the approximate solution obtained with $n$ collocation points. The error norm $v s$. the total number of calculation points $n$ by using the presented method is shown in Figure 3, where the integral in (54) is discretized by the trapezoidal rule. By using only $n=64$ ( 256 calculation points on the whole boundary), we obtain error norm less that $10^{-15}$. The absolute errors $\left|u(z)-u_{n}(z)\right|$ at selected points in the entire domain are plotted in Figure 4. The graph of the approximate solution $u_{n}(z)$ is illustrated in Figure 5.

Example 2 In this example, we consider an unbounded multiply connected region of connectivity 6 (see Figure 6 ). The boundary $\Gamma=\partial G$ is parametrized by 


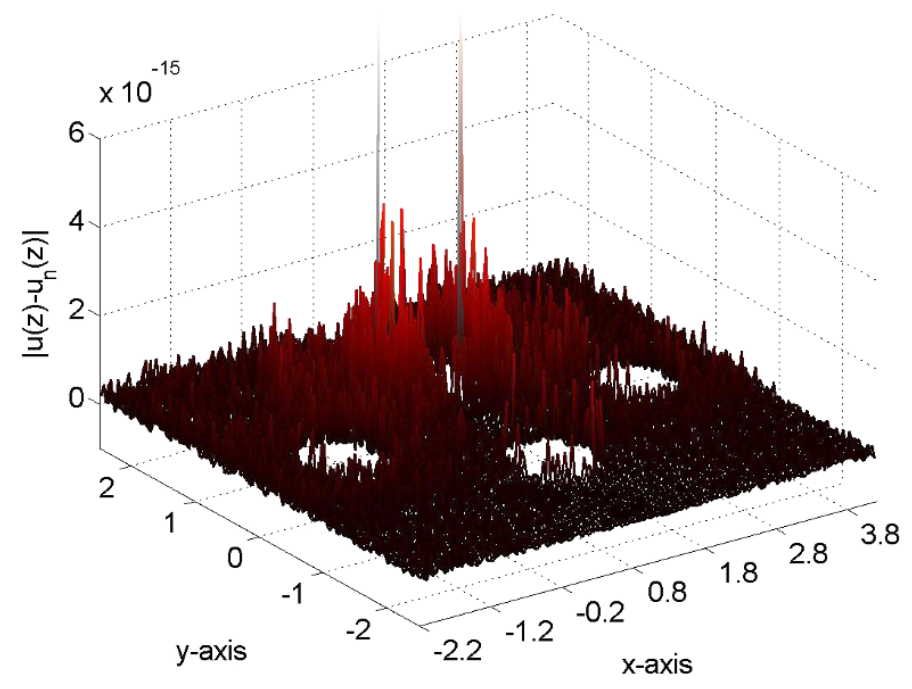

Figure 4 The absolute error for Example 1.

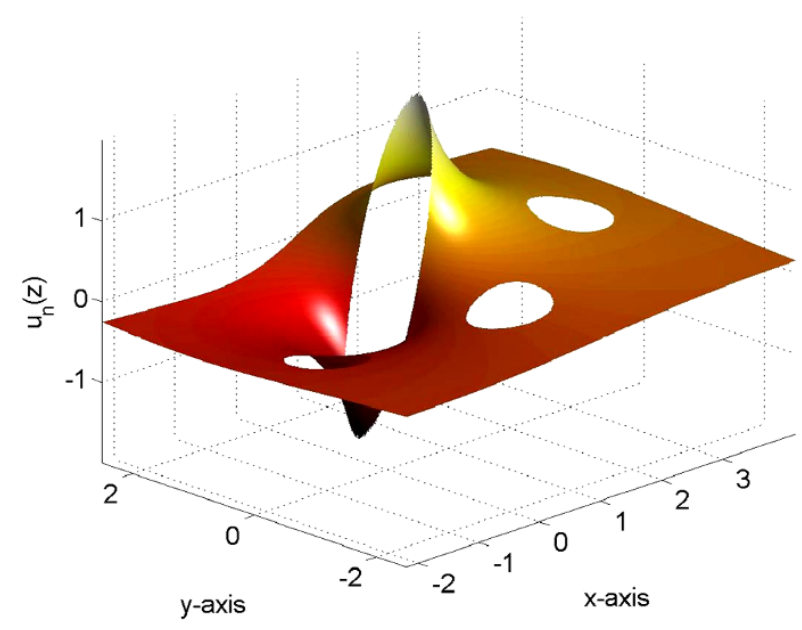

Figure 5 The graph of the approximate solution for Example 1.

$$
\eta_{j}(t)=z_{j}+e^{\mathrm{i} v_{j}}\left(\alpha_{j} \cos t+\mathrm{i} \beta_{j} \sin t\right), \quad j=1, \ldots, 6,
$$

where the values of the complex constants $z_{j}$ and the real constants $\alpha_{j}, \beta_{j}, v_{j}$ are as in Table 1.

The region in this example has been considered in $[3,17,18]$ for the Dirichlet problem and the Neumann problem. In this example, we consider a mixed boundary value problem where we assume that the conditions on the boundaries $\Gamma_{1}, \Gamma_{2}, \Gamma_{3}$ are the Dirichlet conditions and the conditions on the boundaries $\Gamma_{4}, \Gamma_{5}, \Gamma_{6}$ are the Neumann conditions. The functions $\phi_{j}$ in (6b)-(6c) are obtained based on choosing an exact solution of the form

$$
u(z)=1+\sum_{j=1}^{6}(j-7 / 2) \log \left(\left|z-\zeta_{j}\right|^{2}\right)
$$




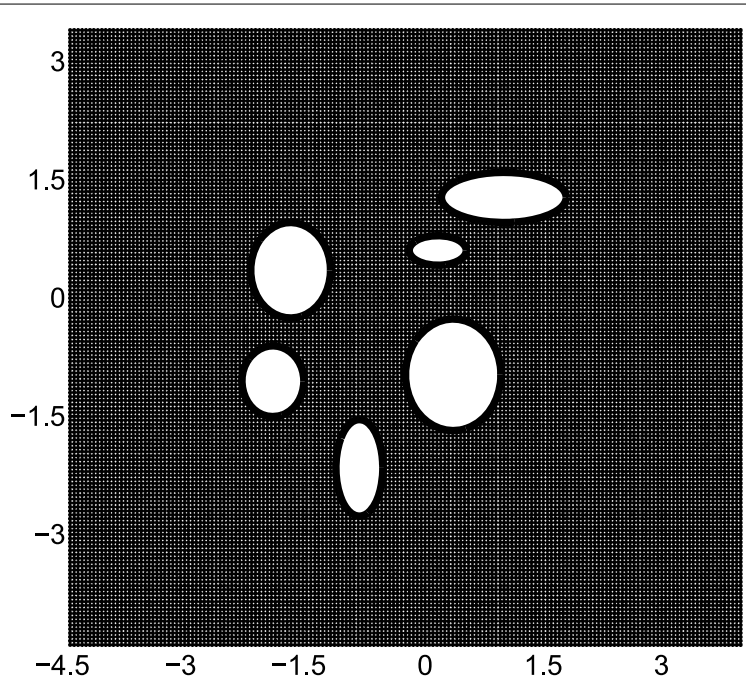

Figure 6 The region for Example 2.

Table 1 The values of constants $\alpha_{j}, \beta_{j}, z_{j}, v_{j}$ and $\zeta_{j}$ for Example 2

\begin{tabular}{lllrlr}
\hline $\boldsymbol{j}$ & $\boldsymbol{\alpha}_{\boldsymbol{j}}$ & $\boldsymbol{\beta}_{\boldsymbol{j}}$ & \multicolumn{1}{l}{$\boldsymbol{z}_{\boldsymbol{j}}$} & \multicolumn{1}{l}{$\boldsymbol{v}_{\boldsymbol{j}}$} & \multicolumn{1}{c}{$\boldsymbol{\zeta}_{\boldsymbol{j}}$} \\
\hline 1 & 0.3626 & -0.1881 & $0.1621+0.5940 \mathrm{i}$ & 3.3108 & $0.10+0.50 \mathrm{i}$ \\
2 & 0.5061 & -0.6053 & $-1.7059+0.3423 \mathrm{i}$ & 0.5778 & $-1.60+0.40 \mathrm{i}$ \\
3 & 0.6051 & -0.7078 & $0.3577-0.9846 \mathrm{i}$ & 4.1087 & $0.30-0.90 \mathrm{i}$ \\
4 & 0.7928 & -0.3182 & $1.0000+1.2668 \mathrm{i}$ & 2.6138 & $0.95+1.20 \mathrm{i}$ \\
5 & 0.3923 & -0.4491 & $-1.9306-1.0663 \mathrm{i}$ & 4.4057 & $-1.85-1.00 \mathrm{i}$ \\
6 & 0.2976 & -0.6132 & $-0.8330-2.1650 \mathrm{i}$ & 5.7197 & $-0.80-2.10 \mathrm{i}$ \\
\hline
\end{tabular}

where the values of the complex constants $\zeta_{j}$ are as in Table 1. For the error, we use the error norm (see Figure 7)

$$
\int_{0}^{2 \pi}\left|u\left(-2.0-0.25 \mathrm{i}+1.5 e^{-\mathrm{i} t}\right)-u_{n}\left(-2.0-0.25 \mathrm{i}+1.5 e^{-\mathrm{i} t}\right)\right|^{2} d t .
$$

The absolute errors $\left|u(z)-u_{n}(z)\right|$ at selected points in the entire domain are plotted in Figure 8 . The graph of the approximate solution $u_{n}(z)$ is shown in Figure 9.

Example 3 This example aims to give an impression how the method works for a problem with an unknown exact solution. We assume that the boundaries of an unbounded doubly connected region are represented as follows (see Figure 10):

$$
\begin{aligned}
& \Gamma_{1}: \eta_{1}=-2-\mathrm{i}+(2+0.5 \cos 5 t) e^{-\mathrm{i} t}, \\
& \Gamma_{2}: \eta_{2}=4-\mathrm{i}+\cos t-3 \mathrm{i} \sin t
\end{aligned}
$$

We assume the Dirichlet condition on the star-shape boundary with Dirichlet data $\phi_{1}(t)=$ 4 , while on the ellipse-shape boundary is the Neumann condition with Neumann data $\frac{\partial u}{\partial \mathbf{n}}=\phi_{2}(t)=-4$. The graph of the approximate solution is illustrated in Figure 11. 


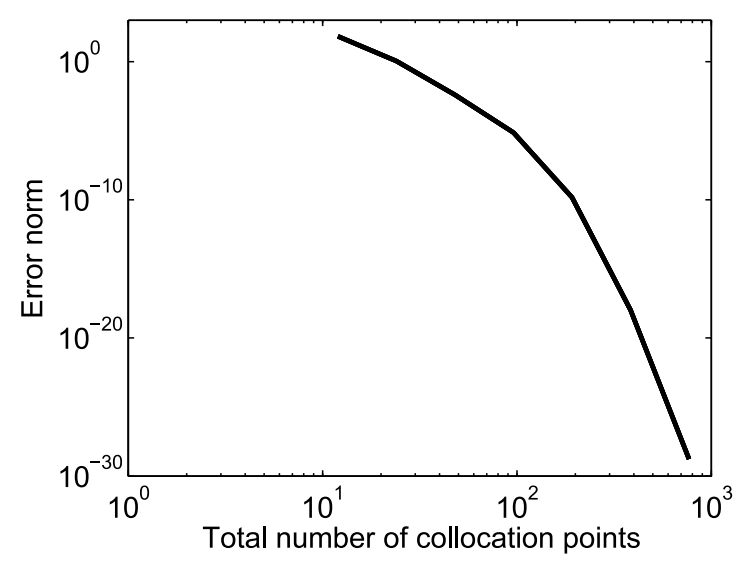

Figure 7 The error norm (56) for Example 2.

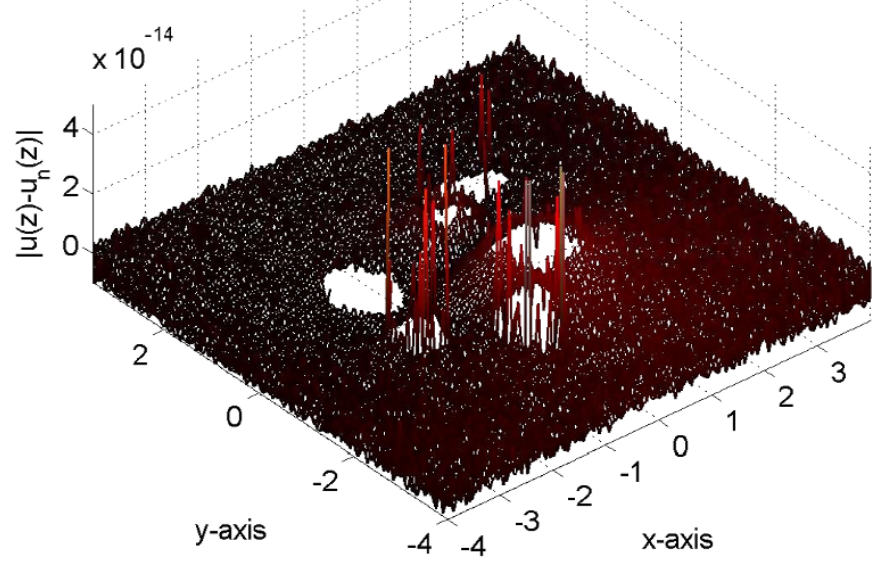

Figure 8 The absolute error for Example 2.

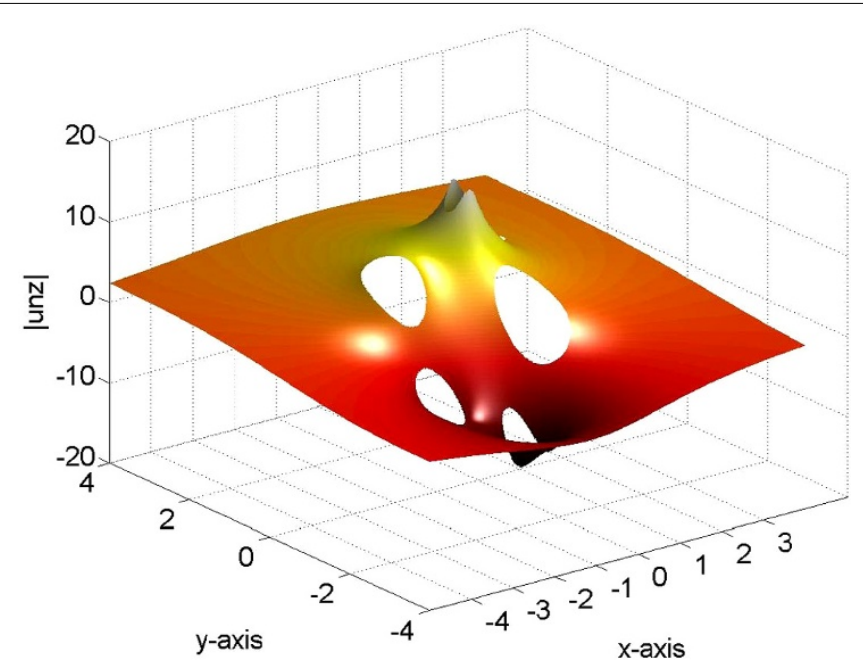

Figure 9 The graph of the approximate solution for Example 2. 


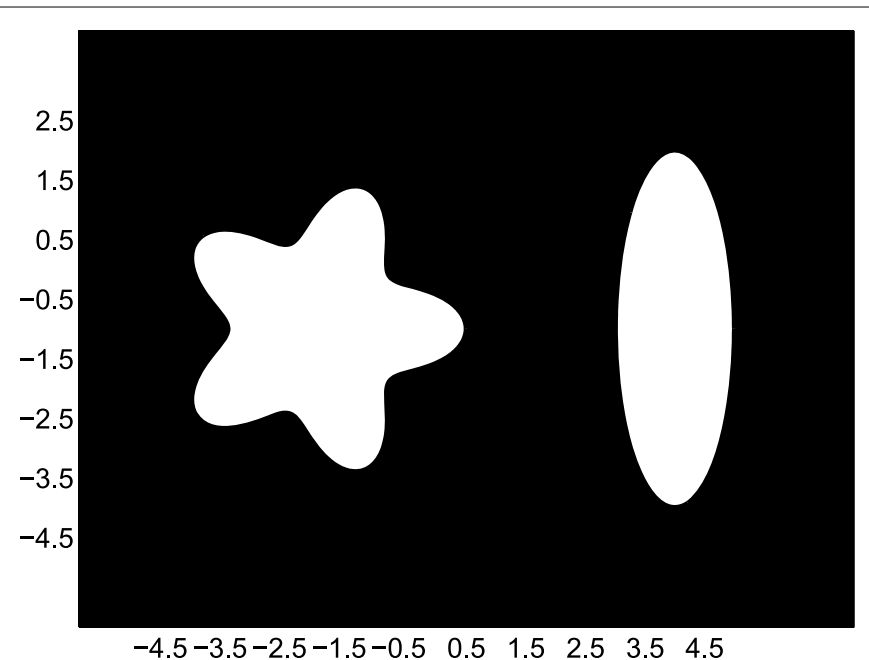

Figure 10 The region for Example 3.

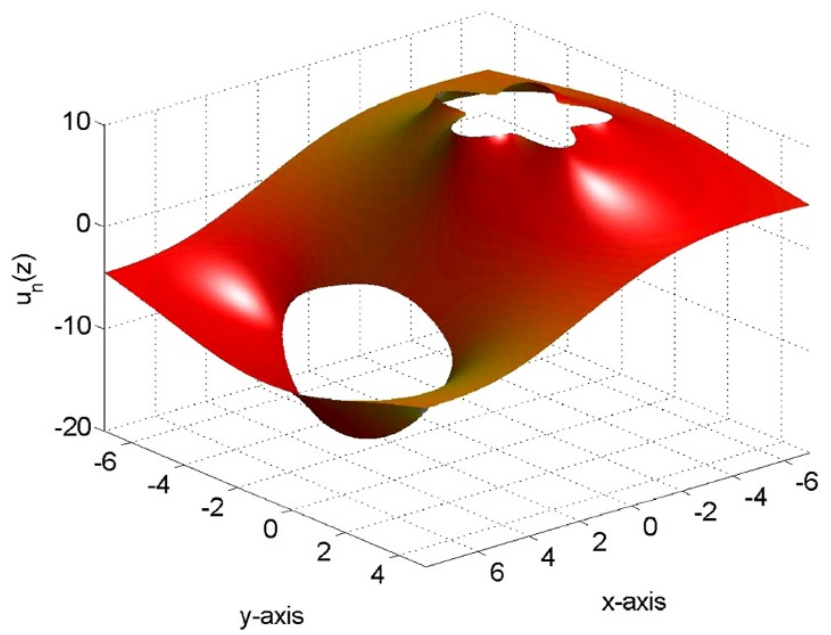

Figure 11 The graph of the approximate solution for Example 3.

\section{Conclusion}

We have constructed a new boundary integral equation with the generalized Neumann kernel for solving a mixed boundary value problem in unbounded multiply connected regions. The generalized Neumann kernel used in this paper is formed with $A(t)=e^{-\mathrm{i} \theta(t)}$ which is different from the ones used in $[1,3]$. Numerical evidences show that Theorem 8 in [3], which claims that the eigenvalues of the generalized Neumann kernel lie in $[-1,1)$, is no longer true for the function $A(t)$ of this paper (see Figures 12 and 13). Three numerical examples are presented to illustrate the accuracy of the presented method. The numerical examples illustrate that the proposed method yields approximations of high accuracy. This paper only applies to the explicitly mixed Dirichlet and Neumann boundary conditions in unbounded multiply connected regions, but the method can be extended to a boundary with both mixed boundary conditions in a boundary component $\Gamma_{k}$. For this case, the function $A(t)$ is discontinuous on $J_{k}$, where $A(t)=1$ on the part of $\Gamma_{k}$ corresponding to the 


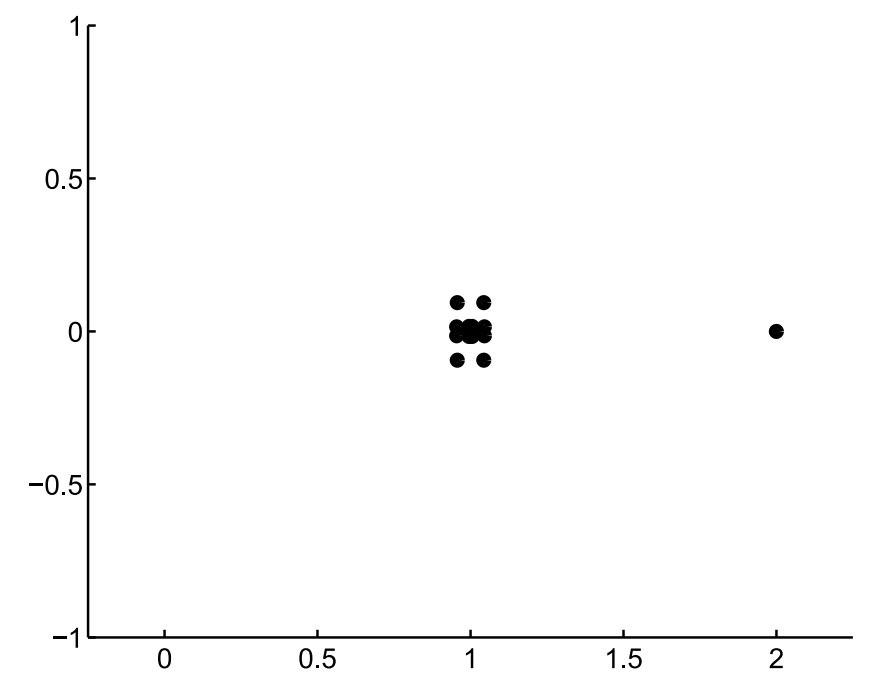

Figure 12 The eigenvalues of the coefficient matrix of the linear systems obtained by discretizing integral equations (45) with $n=\mathbf{2 5 6}$ for the region in Example 1 .

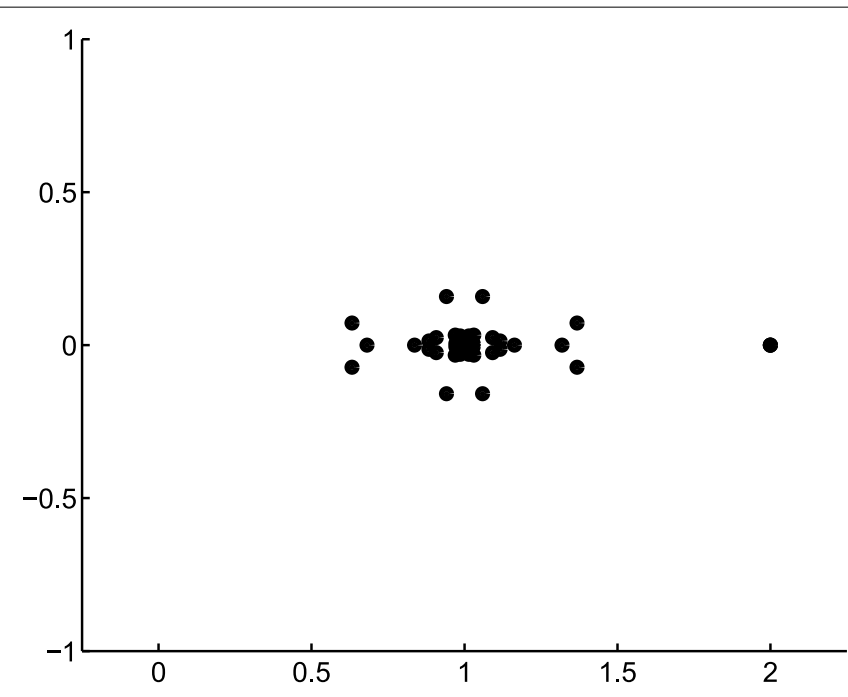

Figure 13 The eigenvalues of the coefficient matrix of the linear systems obtained by discretizing integral equations (45) with $n=\mathbf{2 5 6}$ for the region in Example 2 .

Dirichlet condition and $A(t)=-\mathrm{i}$ on the part of $\Gamma_{k}$ corresponding to the Neumann condition. Hence, the RH problem (42) will be a problem with discontinuous coefficient $A(t)$. Thus, further investigations are required. This will be considered in future work.

Competing interests

The authors declare that they have no competing interests.

Authors' contributions

All authors contributed equally to the manuscript and typed, read and approved the final manuscript.

\section{Author details}

${ }^{1}$ Department of Mathematical Sciences, Faculty of Science, Universiti Teknologi Malaysia, Johor Bahru, Johor 81310 UTM, Malaysia. ${ }^{2}$ UTM Centre for Industrial and Applied Mathematics, Universiti Teknologi Malaysia, Johor Bahru, Johor 81310 
UTM, Malaysia. ${ }^{3}$ Department of Mathematics, Faculty of Science, King Khalid University, P.O. Box 9004, Abha, Saudi Arabia. ${ }^{4}$ Department of Mathematics, Faculty of Science, Ibb University, P.O. Box 70270, lbb, Yemen.

\section{Acknowledgements}

The authors would like to thank the editor and referees for their helpful comments and suggestions which improved the presentation of the paper. The authors acknowledge the financial support for this research by the Malaysian Ministry of Higher Education (MOHE) through UTM GUP Vote Q.J130000.7126.01H75.

Received: 29 September 2012 Accepted: 8 February 2013 Published: 14 March 2013

\section{References}

1. Nasser, MMS, Murid, AHM, Al-Hatemi, SAA: A boundary integral equation with the generalized Neumann kernel for a certain class of mixed boundary value problem. J. Appl. Math. 2012, Article ID 254123 (2012). doi:10.1155/2012/254123

2. Wegmann, R, Nasser, MMS: The Riemann-Hilbert problem and the generalized Neumann kernel on multiply connected regions. J. Comput. Appl. Math. 214, 36-57 (2008)

3. Nasser, MMS, Murid, AHM, Ismail, M, Alejaily, EMA: Boundary integral equations with the generalized Neumann kernel for Laplace's equation in multiply connected regions. Appl. Math. Comput. 217, 4710-4727 (2011)

4. Nasser, MMS: Numerical conformal mapping via a boundary integral equation with the generalized Neumann kernel. SIAM J. Sci. Comput. 31, 1695-1715 (2009)

5. Nasser, MMS: Numerical conformal mapping of multiply connected regions onto the second, third and fourth categories of Koebe's canonical slit domains. J. Math. Anal. Appl. 382, 47-56 (2011)

6. Yunus, AAM, Murid, AHM, Nasser, MMS: Conformal mapping of unbounded multiply connected region onto canonical slit regions. Abstr. Appl. Anal. 2012, Article ID 293765 (2012). doi:10.1155/2012/293765

7. Gakhov, FD: Boundary Value Problem. Pergamon, Oxford (1966)

8. Haas, R, Brauchli, H: Fast solver for plane potential problems with mixed boundary conditions. Comput. Methods Appl. Mech. Eng. 89, 543-556 (1991)

9. Haas, R, Brauchli, H: Extracting singularities of Cauchy integrals - a key point of a fast solver for plane potential problems with mixed boundary conditions. J. Comput. Appl. Math. 44, 167-185 (1992)

10. Nasser, MMS: A boundary integral equation for conformal mapping of bounded multiply connected regions. Comput. Methods Funct. Theory 9, 127-143 (2009)

11. Gonzlez, R, Kress, R: On the Treatment of a Dirichlet-Neumann mixed boundary value problem for harmonic functions by an integral equation method. SIAM. J. Math. Anal. 8, 504-517 (1977)

12. Mikhlin, SG: Integral Equations and Their Applications to Certain Problems in Mechanics Mathematical Physics and Technology. Pergamon, New York (1957)

13. Atkinson, KE: The Numerical Solution of Integral Equations of the Second Kind. Cambridge University Press, Cambridge (1997)

14. Nasser, MMS, Murid, AHM, Zamzamir, Z: A boundary integral method for the Riemann-Hilbert problem in domains with corners. Complex Var. Elliptic Equ. 53(11), 989-1008 (2008)

15. Krommer, AR, Ueberhuber, CW: Numerical Integration on Advanced Computer Systems. Springer, Berlin (1994)

16. Helsing, J, Ojala, R: On the evaluation of layer potentials close to their sources. J. Comput. Phys. 227, $2899-2921$ (2008)

17. Greenbaum, A, Greengard, L, MCFadden, GB: Laplace's equation and the Dirichlet-Neumann map in multiply connected domains. J. Comput. Phys. 105(2), 267-278 (1993)

18. Helsing, J, Wadbro, E: Laplace's equation and the Dirichlet-Neumann map: a new mode for Mikhlin's method. J. Comput. Phys. 202, 391-410 (2005)

\section{Submit your manuscript to a SpringerOpen ${ }^{0}$ journal and benefit from:}

- Convenient online submission

- Rigorous peer review

- Immediate publication on acceptance

- Open access: articles freely available online

- High visibility within the field

- Retaining the copyright to your article 